\title{
Labyrinthe
}

18 | 2004 (2)

La Recherche dans tous ses éclats

\section{L'honnête homme : mérite et pouvoir chez La Bruyère}

\section{Christophe Cervellon}

\section{(2) OpenEdition \\ 1 Journals}

Édition électronique

URL : http://journals.openedition.org/labyrinthe/228

DOI : $10.4000 /$ labyrinthe.228

ISSN : 1950-6031

Éditeur

Hermann

Édition imprimée

Date de publication : 15 juillet 2004

Pagination : 105-121

Référence électronique

Christophe Cervellon, "L'honnête homme : mérite et pouvoir chez La Bruyère », Labyrinthe [En ligne]

18 | 2004 (2), mis en ligne le 24 juin 2008, consulté le 30 avril 2019. URL : http://

journals.openedition.org/labyrinthe/228; DOI : 10.4000/labyrinthe.228

Propriété intellectuelle 


\section{L'HONNÊTE HOMME: MÉRITE ET POUVOIR CHEZ LA BRUYÈRE}

Christophe CERVELLON cervellon@wanadoo.fr

Il est difficile de dire si le mérite est avant tout un concept théologique - puisque c'est en théologie qu'il a connu apparemment ses explicitations les plus complexes, de Tertullien à Luther - ou un concept politique, de Cicéron à la méritocratie républicaine. Il est difficile de dire aussi si la notion de mérite en théologie est passée dans le champ politique, ou si un même concept formel a joué dans ces deux champs, ou si nous sommes encore en présence de deux histoires intellectuelles différentes de l'idée de mérite, l'une proprement théologique, l'autre proprement politique. Une chose est certaine, en tout cas : le XVII ${ }^{\mathrm{e}}$ siècle, si augustinien, si «théologique», est avant le XVIII siècle, eût dit La Palisse, qui devait nous léguer une nouvelle manière de concevoir la légitimité du pouvoir. L'acception théologique du mérite, encore présente chez La Rochefoucauld (1613-1680) laisse la place chez La Bruyère (1645-1696), une génération plus tard, à une conception plus résolument politique du mérite personnel. Tout se passe comme si, sans préjuger du lien entre le théologique et le politique (transformation de l'un dans l'autre ou simple rencontre autour d'un même problème logique: à quelles conditions ce que je suis peut me donner droit à quelque chose), c'était le plan politique qui prenait la première place. Loin de toute théologie morale, nous nous proposons de voir concrètement comment le mérite devient chez La Bruyère moral et politique.

Étrange droit, en vérité, qu'ouvre le mérite, puisqu'on ne peut faire valoir ce droit sans immédiatement démériter, sans paraître intéressé, et pour dire le vrai, au-dessous du mérite dont on s'habille, qui était tout à l'heure le fonds clair de notre être et qui n'est plus - à être ainsi moins réclamé que proclamé - que sa face perceptible et agressive : «Il coûte [dit La Bruyère] à un homme de mérite de faire assidûment sa cour [...]. Celui au contraire qui a une bonne opinion de soi et que le vulgaire 
appelle un glorieux, a du goût à se faire voir.» L'homme de mérite, qui se connaît tel, ne peut se faire connaître, sous peine de devenir «glorieux », c'est-à-dire fier et incommode aux autres. C'est bien ainsi que l'entend Molière, dans Le Bourgeois gentilhomme, III, 12: «Voyezvous, dirait-on, cette madame la Marquise qui fait tant la glorieuse?» La modestie l'enveloppe cependant dans un demi-jour incertain qui le sert ou dessert, selon l'occasion: «La modestie est au mérite ce que les ombres sont aux figures dans un tableau: elle lui donne de la force et du reflet. » Elle l'éloigne, d'une part, trop longtemps des offices dont il est digne, mais prévient, d'une autre part, les dangers que réserve la Cour à chaque instant. Plus qu'il ne la garde, d'ailleurs, c'est la modestie qui garde l'homme de mérite, tant il est vrai que ce ne sera pas l'honnête homme qui la feindra comme la seule ruse moralement permise dans la société des hommes «habiles». À l'image (à l'imitation?) du couvreur, l'homme de cœur est appliqué à bien faire, «pendant que le fanfaron travaille à ce que l'on dise de lui qu'il a bien fait».

De l'homme de mérite, la modestie est ainsi l'essence protectrice, mais également le fonds et le caractère: elle doit signifier, par le refus de tout signe extérieur, ce qui ne doit pas être signifié, et qui ne doit être vécu que dans un silence respectueux, autant du roi comme de nous-mêmes. Le mérite est une réclamation silencieuse: s'il se raconte, il devient comme une protestation vaniteuse - il perd son droit, qui était droit réel à quelque chose - et semble une protestation vaine: il perd encore ce sur quoi était fondé le droit formel qu'il avait de réclamer. Barthes aurait sans doute dit que le mérite est pour La Bruyère une métaphore impossible, un caractère qu'on ne peut pas développer, une valeur immédiatement dévaluée dans le démérite dès lors qu'elle se fait savoir dans un savoir-faire social. Et à vrai dire, le mérite n'est pas un caractère, une manière de pointer dans la conduite ce qui est traits, marques, fausses singularités, comme autant de signifiants-effets d'un signifié-cause, qui produit en effet des signes comme autant de raisons de voir et de faire, et comme autant de raisons de ces raisons. Le mérite est discret, au hasard de ne jamais être reconnu: «Vous dites qu'il faut être modeste, les gens bien nés ne demandent pas mieux : faites seulement que les hommes n'empiètent pas sur ceux qui cèdent par modestie, et ne brisent pas ceux qui plient.»

Mais si le mérite n'est pas un caractère, comme le sont la pauvreté, l'étourderie, l'hypocrisie, ou la manie, c'est aussi que dans l'ordre de 
la nature, où l'on peut se rendre digne ou indigne des emplois, l'homme de mérite est l'homme tout entier, puisqu'il est l'homme qu'il faut être, cependant que dans l'état social, qui est l'ordre réel des emplois, les hommes d'un mérite personnel constituent une classe à part, où leur mérite peut n'être ni reconnu ni employé. Ni catégorie sociologique (des Femmes), ni catégorie métaphysique (de l'Homme), ni catégorie psychologique (du Cour $)$, le chapitre «Du mérite personnel» relève un peu aussi de toutes ces catégories, comme si le discours du mérite au $\mathrm{XVII}^{\mathrm{e}}$ siècle ne pouvait être autre chose qu'une économie de la transgression catégorielle.

\section{Position du problème}

Mais qu'est-ce qu'un droit qu'on ne peut faire valoir, qu'on ne peut exiger, à quoi on ne peut prétendre sans présomption - en anticipant, par la possession d'un titre, sur une propriété qui appartient, avec droit d'user et de mésuser, à un autre - , sinon un droit qui vaut sans exister, qui a une existence axiologique, à la condition de ne pas entraîner une reconnaissance mécanique : un droit formel de réclamer qui n'a pas de droit réel à obtenir? Le roi ne nous doit rien, et c'est ce que l'on appelle, entre autres raisons, le pouvoir absolu. D'ailleurs, l'homme de mérite n'a-t-il pas fait simplement ce qu'il devait faire, et satisfait en somme aux devoirs de sa charge ? Qu'y a-t-il de méritoire à accomplir les affaires auxquelles nous sommes étroitement obligés? N'est-il pas commandé à tous les bons sujets «d'employer les premières années de leur vie à devenir tels par leurs études et par leur travail que la république elle-même eût besoin de leur industrie et de leurs lumières »?

En accomplissant notre devoir, nous ne nous relevons en rien : nous pouvons simplement ainsi espérer rendre ce qui est justement dû au souverain. C'est à lui, s'il le juge bon, de relever plus spécialement tel ou tel - dispunctio meriti, comme aurait dit Tertullien, parlant d'un autre Dieu - qui n'a pourtant fait rien d'autre que ce qu'il était étroitement tenu de faire; et s'il s'est enfin distingué, c'est que les autres ont démérité. Tous doivent également retirer leur billet, et s'acquitter ainsi envers la société, et le roi, de la dette que chacun a originellement acquise par le simple fait de naître français et sujet: «Nous devons travailler à nous rendre très dignes de quelque emploi : le reste ne nous regarde point, c'est l'affaire des autres. » 
Si le souverain peut et doit reconnaître le mérite, si la res publica doit précisément rendre publique et effective la valeur objective des individus, contre toutes les valeurs d'exposition que monnaie la Cour, la monarchie n'est pas une méritocratie, et la nécessité morale qui incombe au roi d'employer les hommes capables n'est aucunement un système politique - qu'à ce défaut on ne peut pas même dire qu'elle préfigure. La Bruyère n'est pas un révolutionnaire: dans l'ombre de l'ordre monarchique, le seul ordre qui défende du despotisme, ne grandit pas un «collectif» d'hommes méritants qui, ne pouvant réclamer pour eux leur droit au souverain, demanderont un jour la souveraineté pour le peuple. Il n'y a pas de contrat social qui fixe ce que le souverain nous doit. Certes, il y a bien un «retour de devoirs du souverain à ses sujets, et des sujets au souverain». Mais si le souverain nous doit politiquement la justice et la sécurité, il ne nous doit que moralement la reconnaissance de notre valeur personnelle, dans la mesure même où nos services les plus dévoués ne sont jamais que les corrélatifs de ces seules obligations «indispensables »-justice et sécurité, bonté et protection - que le souverain a envers nous. C'est parce que nous avons le devoir de bien servir, que le roi a le devoir de bien gouverner et de bien protéger. Mais si bien servir est un devoir qui nous donne des droits politiques, il ne donne pas celui d'être bien employé : il convient au roi, selon une simple nécessité de convenance, d'unir la morale à l'efficacité en donnant les bons postes aux plus dignes. Mais de même que gouverner efficacement n'est pas gouverner justement, employer ceux qui le méritent aux places qu'ils méritent dépasse l'ordre de la stricte justice, même distributive. Les droits qu'ouvre ainsi le mérite ne se réclament pas, ni pour soi, au hasard d'être jugé glorieux, ni pour les autres, au risque de réclamer injustement pour une catégorie d'hommes ce qui n'est point politiquement dû, et de contester, au nom d'une méritocratie virtuelle, le régime qui seul existe en fait et de droit: «Ce qu'il y a de plus raisonnable et de plus sûr, c'est d'estimer [la forme de gouvernement] où l'on est né la meilleure de toutes et de s'y soumettre.»

\section{Le possible chez La Bruyère}

Comment comprendre, dans ces conditions, ce que veut dire exactement La Bruyère lorsqu'il affirme que la louange s'applique aussi justement à l'acte possible qu'à l'acte réel? «Tels peuvent être loués de ce qu'ils ont fait, et tels de ce qu'ils auraient fait.» Car si tel peut 
être loué de ce qu'il pourrait faire, reste qu'au moment même où l'acte méritoire est possible il n'a pas, comme on l'a vu, un droit légal à être. C'est sans doute dans l'exigence d'une susception à l'ordre seul légitime et légal, de ce qui est possible en fait - l'acte méritoire - mais qui n'a pas un droit opposable à être, que se dissimule la philosophie politique si complexe de la Bruyère: d'une part, la réalité politique, entièrement justifiée en droit - ou légitime; d'autre part, un ordre différent possible, où les postes sont autrement répartis, où d'autres actes sont produits par d'autres hommes, mais un ordre amendé qui n'est que possible, qui n'a de pas de droit à l'existence à faire valoir, et dont l'existence possible, nécessairement silencieuse, n'est pourtant pas un pur rien: car si l'existence de l'acte méritoire n'est que possible, et n'a pas un droit légal à exister, ce possible a cependant une forme d'existence. Ce n'est pas parce qu'on n'a pas un droit à exister effectivement qu'on n'existe pas formellement, et cette forme de reconnaissance qu'est la louange - reconnaissance qui n'engage politiquement à rien, qui n'a aucun contenu juridique - s'adresse autant à l'existence effective qu'à l'existence formelle de l'acte méritoire: «[L'homme de mérite est] admirable même et par les choses qu'il a faites, et par celles qu'il aurait pu faire ${ }^{1}$.»

La Bruyère ne dit jamais ce qui sera affirmé au XIX ${ }^{e}$ siècle par Jules Lagneau: "Ce qui méritera d'être sera»; il dit plutôt que ce qui mérite d'être est déjà suffisant pour qu'on puisse le reconnaître, et le louer, le reconnaître privément, sinon publiquement. Le mérite existe comme une chose, un être en acte, une res meritans, quelque chose qui a une quantité de réalité où il peut entrer, comme c'est le cas chez Celse, du peu, du moins et du beaucoup, parce que le possible peut être plus ou moins possible. On peut ne pas en avoir assez, et à vrai dire, on n'en a jamais assez. Le mérite est moins dans la réalisation toujours retardée d'un possible qui n'existe pas (valeur ou idéal de la personne que l'on voudrait être, ou de l'acte que l'on devrait faire), que dans l'effectuation possible de ce qui existe déjà : l'homme de mérite, qui pourrait faire des actes méritoires, qui ne sont que possibles, ne laisse pas d'exprimer continuellement un mérite actuel. Comment, aussi bien, comprendrions-nous une louange qui s'adresse non pas tant à ce qui n'est pas encore, qu'à ce qui n'est pas et ne peut pas être? 
À peine la réalité du mérite ressortit-elle ainsi à la catégorie du devoir-être, à l'effort commun de la volonté et du jugement par lequel est posée, avant que réalisée, l'identité de ce que l'on juge bon d'être et du bien voulu par ce jugement, ou du rôle que l'on juge bon de tenir avec l'emploi dont on se croit digne: faute d'une absolue droiture, on s'exposerait alors à jouer un rôle non seulement devant les autres mais devant soi-même: "Certains hommes, contents d'eux-mêmes, de quelque action ou de quelque ouvrage qui ne leur a pas mal réussi, et ayant ouï dire que la modestie sied bien aux grands hommes, osent être modestes [...]: semblables à ces gens d'une taille médiocre qui se baissent aux portes de peur de se heurter.» Au contraire, l'acte méritoire a beau ne pas exister effectivement, faute d'occasion, tout laisse voir qu'il aurait pu exister, car sa possibilité est comme inscrite dans la réalité même de l'être, et dans les formes différentes, des plus basses aux plus hautes, que prend cette réalité. Le possible n'est pas qu'un être en puissance, puisqu'il s'exprime, car «il n'y a rien de si délié [nous est-il dit dans le chapitre «Du mérite personnel»] de si simple, et de si imperceptible, où il n'entre des manières qui nous décèlent. Un sot ni n'entre, ni ne sort, ni ne s'assied, ni ne se lève, ni ne se tait, ni n'est sur ses jambes, comme un homme d'esprit». Et si le possible a ainsi moins besoin pour être d'être réalisé, que de se laisser voir, ce philosophe dont la critique sociale semble parfois anticiper sur celle du siècle suivant, peut écrire sans contradiction ni flagornerie que chez les «enfants des dieux», «le mérite [...] devance l'âge». Non point précisément que le sang tire les «enfants des dieux » des règles de la nature, mais le mérite qui n'est que possible ne laisse pas d'être avec l'actualité d'une chose. Le grand n'est pas un cas particulier dans l'ordre de la nature, le représentant d'une sur-nature qui le ferait suivre une loi propre, mais l'exemple privilégié (à tous les sens du mot), qualitativement distinct et non pas spécifiquement différent, d'une loi générale, selon laquelle, dans l'ordre du mérite, le possible ne laisse pas que d'avoir une forme d'existence, quand même l'acte méritoire n'a pas et même n'a jamais eu lieu.

Car l'estime va à l'individu, et non pas à ce qu'il porte - titre, charge ou nom, qui ne mettent guère au jour ce qu'il est vraiment. Et, d'ailleurs, qu'est-il vraiment? Face à l'évidence des conduites, qui sautent aux yeux, La Bruyère renonce aux certitudes complexes, qui vont à l'homme. Disons mieux: ce que l'être est vraiment peut parfois être 
rendu impossible par la vie, et qui est né doux peut devenir «dur et épineux ». Du possible au réel, la conséquence est rarement bonne, ce qui définit à la fois le drame humain et le drame politique. Pire encore: le possible, rendu impossible par la vie, garde encore une forme d'existence, et qui est né doux ne vit pas sa douceur au passé : la dureté n'a pas exclu la douceur, comme un caractère chasse un autre, en l'éliminant ou en le rejetant dans la mémoire. C'est parce que le possible a une existence que, même rendu impossible, il ne disparaît pas entièrement comme s'il n'avait pas été; et pourtant, en y réfléchissant bien, il est vrai qu'il n'a jamais été, puisque c'est bien «pendant toute sa vie» que «tel a vécu [...] chagrin, emporté [...] qui était né gai, paisible...». "Ainsi tel homme au fond et en lui-même ne se peut définir: trop de choses qui sont hors de lui l'altèrent, le changent, le bouleversent; il n'est point précisément ce qu'il est ou ce qu'il paraît être.» La Bruyère ne veut évidemment pas dire ici qu'on ne peut définir un homme $a$ priori ou avant qu'il ne vive concrètement entre les autres hommes ( «le corps est étendu», «tel homme est tel»); mais il ne dit pas non plus la banalité contraire, qu'on ne peut guère que caractériser l'homme, c'està-dire le peindre, par des propriétés («le corps est lourd», «tel homme marche, court, réclame ainsi ») qui supposent l'expérience de la vie et les relations sociales.

Il dit simplement qu'il y a des possibles qui, même rendus intrinsèquement impossibles, ne sont pas exactement rien, condition qui ne peut être exactement peinte - sinon, dans le cas exceptionnel du mérite, selon le clair-obscur de la modestie - ni même exactement pensée : il y a quelque chose qui nous rend étrangers à nous-mêmes, mais qui n'est pas autre que nous-mêmes. Et cette étrangeté ne vient pas du recul que nous pouvons toujours prendre sur nos actions, de ce pouvoir de nous regarder comme autres, de nous voir ridicules ou sinistres dans les jeux de la Cour, joint que nous avons conscience qu' aucune imposture n'est jamais parfaite, ni pour les autres, ni pour nous-mêmes ; mais cette étrangeté résulte du fait qu'un possible avorté n'est jamais un possible mort: «Tout est étranger dans l'humeur, les mours et les manières de la plupart des hommes.» Il y a un rythme intérieur, fait de possibles plus ou moins réalisés, qui nous fait sentir à nous-mêmes par un système de dissonances intimes, et qui nous individue obscurément en nous, sans nous et malgré nous, à nos propres yeux comme aux regards des autres. 
Ce rythme intérieur de possibles plus ou moins avortés, plus ou moins vivants, qui mêle inextricablement dans un même cœur douceur et dureté, ne rappelle aucunement la cadence de La Rochefoucauld, ce rapport proportionnel de l'être à l'air ou à l'allure qu'il prend, qui est susceptible de fonder un ordre d'apparences vraies. La Bruyère apprécie ainsi l'énumération bizarre d'adjectifs souvent proches, comme si une énumération avait moins pour tâche de multiplier des points de vue que de dessiner une ligne intensive de l'être humain: mélange d'outrance et de faiblesse, où tout se mêle et se contredit. Car cette ligne est aussi très souvent une ligne brisée. La Bruyère excelle loin à loin dans l'art du bathos, qui est celui de reprendre en un seul mot ce que l'on prétendait dire en dix. Un portrait, ce n'est peut-être pas tant une métaphore, comme le pense Barthes, ou l'opération logique qui consiste à abstraire la généralisation qu'un cas particulier à lui tout seul enveloppe: ce n'est ni la caricature du vicieux ni l'exemplification et l'amplification d'un vice, mais l'opération graphique qui consiste à schématiser une ligne individuelle, et à l'infléchir selon des possibles dominants. Reste l'essentiel: le possible n'est pas un rien, puisqu'il est plus ou moins intrinsèquement possible, même rendu improbable par les nécessités extérieures de la vie et de la nature; et le possible n'est pas rendu impossible par l'actualisation du possible inverse: la dureté n'élimine pas la douceur qui, à dire vrai, l'accompagne, puisqu'elle entre elle aussi dans ce qu'est l'être "épineux», pour qu'il puisse être dit, se paraître et nous paraître, étranger à lui-même. Nous essaierons de montrer plus loin combien ces remarques très générales sur le possible chez La Bruyère sont importantes pour comprendre la question du mérite.

Mais puisque l'acte méritoire ne se signifie pas, puisqu'il n'est pas encore, s'il est possible, ou qu'il n'est plus, s'il est passé, reste que le mérite désintéressé, qui se contente de lui-même, doit se laisser voir dans le clair-obscur de la modestie, et trouver ainsi son expression adéquate. L'homme de mérite aura donc deux contraires : le glorieux (celui qui cherche maladroitement à exprimer le mérite) et l'habile (celui qui n'a aucun mérite à exprimer, parce qu'il n'a aucune droiture).

L'habile intéressé et l'homme de mérite se ressemblent comme chien et loup à la tombée de la nuit. Auquel des deux s'adresse en effet le jugement suivant? «Il est souvent plus court et plus utile de cadrer aux autres que de faire que les autres s'ajustent à nous. » Qu'un habile 
homme, qui «cache ses passions» et «entend ses intérêts» applique cette maxime, et c'est l'hypocrisie qui a trouvé sa formule. Au contraire, si la maxime concerne l'homme de bien, c'est une philosophie de la politesse, qui «fait paraître l'homme au dehors tel qu'il devrait être intérieurement», qui est ici dangereusement résumée. Selon que, dans l'obscure clarté des jeux de Cour, l'on pratique la politesse avec intérêt ou désintérêt, on est ou bien blanc ou bien noir. Comme le dit La Bruyère, «le motif seul fait le mérite des actions des hommes, et le désintéressement y met la perfection ». Le mérite seul justifie l'ambition, en même temps qu'il justifie de son désintéressement: "Un honnête homme se paye par ses mains de l'application qu'il a à son devoir par le plaisir qu'il sent à le faire, et se désintéresse $e^{2}$ sur les éloges, l'estime et la reconnaissance qui lui manquent quelquefois.»

De même, la vanité trouve avec La Bruyère un concept clair: puisque le mérite possible, n'étant pas rien, travaille l'allure générale de la personne - s'exprime -, sera dit vaniteux celui qui cherchera à signifier par le dehors le mérite qui, ainsi proclamé, s'abîme dans l'irréalité. Signifier le possible, c'est manifester son irréalité actuelle; le mérite ne se dit ni se s'expose, car il est trop inquiet pour peser aux autres. Le vaniteux renverse la relation toujours délicate qui lie dans la conscience du moi et dans celle des autres la valeur objective et subjective de l'individu. L'homme de mérite fonde son contentement sur la valeur objective d'un possible qui n'est pas rien; l'homme vaniteux fonde son contentement sur la valeur subjective prêtée à la réalité de sa personne qui est pour lui tout le possible, de même que Ménippe, «incapable de savoir jusqu'où l'on peut avoir de l'esprit, croit naïvement que tout ce qu'il en a est tout ce que les hommes en sauraient avoir: aussi a-t-il l'air et le maintien de celui qui n'a rien à désirer sur ce chapitre, et qui ne porte envie à personne». Il est vrai que l'homme de mérite comme le vaniteux peuvent ne rien désirer, mais pour des raisons exactement contraires, et le portrait de Ménippe est là encore bien à sa place dans «Du mérite personnel»: l'un se contente de son possible, parce qu'il sait qu'il n'est pas rien, qu'il est un «fonds», comme le dit La Bruyère; l'autre se contente de sa réalité, parce qu'il la croit seule possible. L'un dit: ce que je peux être est réel, et n'envie donc pas tant une réalisation qui dépend d'autrui; l'autre affirme: ce

2. Nous soulignons. 
que je suis est tout ce qu'on peut être. Le vrai mérite est dans le possible, qui s'exprime ou se laisse voir naturellement, parce qu'il a une valeur; le faux mérite est dans l'être qui nie le possible, et c'est bien là pour La Bruyère la définition psychologique de la vanité, et la définition philosophique de l'apparence.

Le véritable homme de mérite est celui qui ne demande rien, pas même l'occasion de manifester son mérite; celui qui a, en somme, assez de «fermeté et une grande étendue d'esprit pour se passer des charges et des emplois, et consentir ainsi à demeurer chez soi et à ne rien faire [...]; [qui] a assez de mérite pour jouer ce rôle avec dignité, [et] [...] assez de fonds pour remplir le vide du temps ». Et cela ne fait que confirmer l'idée fondamentale que nous cherchons à développer: La Bruyère voit le possible comme une réalité à la fois morale et sociale, et puisque la réalité du possible n'est pas une chimère, elle nous satisfait psychologiquement, et nous contente quand, faute d'occasion, nous ne satisfaisons socialement à aucune charge. Nous devons nous rendre dignes de quelque emploi, le reste ne nous concerne en rien; et qui a l'étoffe d'un Trophime peut bien se passer d'être évêque. Le mérite est une chose qui existe, fût-ce sous forme de possible : être vraiment, c'est-à-dire être un homme de mérite, c'est aussi bien faire et pouvoir faire, que pouvoir ne rien faire. Car ne rien faire, pour l'homme de mérite, n'est rien moins que ne pas travailler: "Il faut en France beaucoup de fermeté et une grande étendue d'esprit pour se passer des charges et des emplois. Personne presque n'a assez de mérite ${ }^{3}$ pour jouer ce rôle avec dignité [...]. Il ne manque cependant à l'oisiveté du sage qu'un meilleur nom, et que méditer, parler, lire et être tranquille, s'appelât travailler. » Mais réciproquement, seul celui qui peut ne rien faire a suffisamment de possible en lui pour se contenter de lui-même (ce qui est tout le contraire de la fausse modestie ou de la vaine gloire), et ne pas rechercher dans l'agitation les emplois et les divertissements.

\section{Mérite congru et mérite condigne}

On pourrait formuler ainsi la loi dynamique du mérite: ne mérite que celui qui fait plus que satisfaire, plus que ce qui lui est demandé, lors même qu'il ne peut faire plus, lors même qu' on ne le lui demande d'une manière ou d'une autre, c'est-à-dire qu'on ne lui donne l'occasion de

\footnotetext{
3. Nous soulignons.
} 
faire plus que ce qu'il doit faire. Mériter, c'est d'abord ne pas démériter (suivant l'acception négative du concept de mérite) et ensuite satisfaire toujours davantage (ce qui donne au concept de mérite une positivité à tout le moins relative). Il y a mérite non pas quand on fait plus que satisfaire (puisqu'on ne peut pas faire autre chose que satisfaire, que ce sont même les devoirs auxquels nous sommes étroitement obligés qui nous pèsent le plus, et qu'il est déjà beau de ne pas démériter), mais lorsque l'on satisfait toujours plus, c'est-à-dire lorsqu'on a toujours plus d'occasions de satisfaire, occasions qu'il faut bien, d'une manière ou d'une autre, réclamer. Et dans cette dynamique, on ne voit jamais vraiment quand le mérite puisse cesser d'être la réalité ou la valeur objective d'un simple possible qui demande silencieusement au souverain une occasion nouvelle d'être mis en oeuvre.

On ne peut pas signifier un acte qui n'a pas eu lieu et dont on réclame l'occasion, puisqu'en signifiant quelque chose qui n'est pas, on signifie vainement le rien, ce qui est la vanité même; on ne peut pas faire valoir une compétence qu'on n'a pas encore employée, ni exercée, puisqu'il faut faire ses preuves: or, l'homme de mérite prétend toujours à une charge plus lourde et nul ne sait s'il satisfera à de nouveaux devoirs : «Un homme de mérite qui est en place n'est jamais incommode aux autres par sa vanité : il s'étourdit moins du poste qu'il occupe qu'il n'est humilié par un plus grand qu'il ne remplit pas, et dont il se croit digne.» Réclamer l'occasion d'exercer ses capacités, n'est-ce pas anticiper vainement sur l'avenir? Qui nous assure, sinon l'amour-propre, qui est si flatteur et déceptif, que nous ne sortirons pas des justes mesures que la nature a posées devant nos ambitions légitimes? Je ne suis pas juge certain de mon mérite personnel et du mérite d'autrui: «Le monde est plein de gens qui, faisant intérieurement et par habitude la comparaison d'eux-mêmes avec les autres, décident toujours en faveur de leur propre mérite, et agissent conséquemment. » N'est-ce pas alors au souverain de reconnaître le vrai mérite, c'est-àdire par un même geste de le récompenser? Ne faut-il pas un juge du mérite, qui doit avoir le mérite si rare d'apercevoir et de relever le mérite d'autrui ? Car s'il est vrai que rien ne nous est dû, le souverain ne se doit-il pas à lui-même de ne pas récompenser incongrûment le faux mérite? Ou encore ne se doit-il pas d'adjoindre à la dignité de l'homme les dignités des charges qu'il mérite, selon ce passage qui du singulier au pluriel marque la transition, dans le nombre et le rythme 
secret de la personne riche et pauvre de ses possibles, de l'être aux apparences parfois creuses d'une silhouette psychologique et sociale y attachée. Ne faudrait-il pas enfin que la gradation sociale des postes correspondît à la graduation des possibles, ou aux capacités plus ou moins grandes de chacun?

Il serait peut-être judicieux, pour comprendre La Bruyère, de rappeler ici la distinction que font les théologiens entre mérite condigne, mérite congru faillible et mérite congru infaillible. Il est vrai que le roi ne nous doit rien, qu' aucune récompense ne nous est légitimement due, dès lors que nous n'avons fait que ce que nous devions faire, et qu'on ne peut, en l'espèce, rien réclamer de la justice d'autrui. Si un homme de cœur se distingue dans une bataille, il n'a fait que remplir les devoirs de sa charge, de même que les couvreurs doivent couvrir les clochers les plus hauts, parce que tel est leur métier et qu'on le leur demande. Et si toute peine mérite salaire, elle ne mérite rien d'autre que le salaire justement prévu: c'est la condignité. La difficulté est de satisfaire, et les difficultés les plus extrêmes, comme le risque de mort, sont comprises dans le service.

Cependant, ne doit-on pas prévoir pour le bon couvreur ou le bon soldat une récompense qui puisse s'ajouter au salaire seul prévu? N'est-il pas convenable que certains actes courageux appellent une récompense, non pas de la justice du roi, mais de sa libéralité, puisque de tels actes n'engendrent aucun droit, étant dus ? C'est la bonté du roi qui peut reconnaître le mérite de congruité, et récompenser par un honneur ou une dignité ce qui, à prendre les choses absolument, n'avait pas de droit à récompense. Si le soldat fuit, il démérite, mais s'il se fait tuer, qu'a-t-il fait d'autre que ce qu'il devait faire? Et pourtant ne pas le décorer à titre posthume, n'est-ce pas aussi lui manquer, et sinon commettre une injustice, à tout le moins lui faire injure ? Le mérite congru n'est pas une fiction face au mérite condigne, puisque nous sentons spontanément que ne pas honorer certains actes, qui n'ont aucun droit en stricte justice, n'est pas tout à fait juste. Le bon soldat n'est pas absolument digne d'une récompense, pour ce qu'il n'est pas indigne de sa solde, mais il est bon et convenable qu'il en reçoive une de la libéralité du prince.

D'une manière semblable, Hobbes remarque dans le Léviathan que, dès lors que l'organisateur d'une course a promis une récompense à qui gagnera l'épreuve, il la lui doit infailliblement. Certes, le vainqueur 
n'a pas un mérite de condigno à faire valoir sur la promesse faite, et ce pour trois raisons : premièrement, parce que la récompense peut dépasser la valeur réelle des efforts fournis; deuxièmement, parce que tous les participants ont fait le même trajet et qu'ils ne sont pas tous également récompensés; troisièmement, parce que c'est de la seule libéralité de l'organisateur que la récompense dépend. Le coureur arrivé premier a couru le plus vite possible, comme les autres, et c'est à cela seul qu'il s'était engagé (on ne peut pas s'engager à gagner...). En somme, si l'on ne devait récompenser que le seul engagement du coureur (courir le plus vite possible), le gagnant n'aurait pas plus de droit que le perdant: tous ont fait ce qu'ils s'étaient engagés à faire. L'organisateur s'est cependant lié par une promesse à récompenser infailliblement celui qui ferait mieux que les autres, et prévaudrait; et s'il ne le faisait pas, c'est l'engagement de tous les coureurs à courir le plus vite possible qui n'aurait plus de raison d'être. Dès lors que l'on promet que celui qui fera ce qu'il a à faire mieux que les autres aura droit à une récompense, quand bien même il ne mérite pas son simple salaire (car faire mieux que les autres n'est pas nécessairement faire parfaitement, ni satisfaire), il est non seulement convenable mais moralement nécessaire d'accorder la récompense; et l'on parlera alors de mérite congru infaillible.

Ainsi Hobbes présente-t-il dans la théorie du Contrat exposée dans le Léviathan le mérite congru infaillible - qui ne génère aucun droit, même s'il appelle moralement et nécessairement une récompense - et le mérite condigne, qui est le mérite au sens strict: "Celui qui dans un contrat s'exécute le premier est dit mériter ce qu'il doit recevoir du fait de l'exécution de l'autre, et il le reçoit comme un dû. De même, quand un prix est proposé à beaucoup de gens et doit être donné seulement à celui qui le gagnera [...], bien que ce soit un libre don, le fait cependant de gagner est mériter, avoir son dû, car le droit est transféré par le fait de l'institution du prix [...], mais il y a entre ces deux sortes de mérite, cette différence que, dans le contrat, je mérite en vertu de ma propre puissance et du besoin du contractant, tandis que, dans le cas du libre don, je me trouve mériter seulement du fait de la générosité de celui qui donne. Dans le contrat, je mérite vis-à-vis du contractant qu'il se désaisisse de son droit; dans le cas du libre don, je ne mérite pas que celui qui donne se désaisisse de son droit, mais lorsqu'il s'en est dessaisi, je mérite que ce droit m'appartienne plutôt qu'à un autre. Et 
c'est là, je pense, ce que signifie la distinction des Écoles entre meritum congrui et meritum condigni. »

Nous ne sommes pas avec le souverain en situation de contractant, et nous ne saurions avoir, vis-à-vis de lui, aucun mérite condigne à faire valoir. Nous n'avons pas de droit sur le souverain ou la république, et en ce sens le roi, en stricte justice, ne nous doit rien. S'il a des devoirs envers nous, il n'a pas celui de nous faire valoir. En revanche, le roi ne doit-il pas à sa propre personne de faire un bon usage de la libéralité avec laquelle il accorde les places et les dignités, et de ne pas récompenser incongrûment le faux mérite ? D'un côté, on ne peut pas reprocher au roi de ne pas récompenser le mérite; mais, d'un autre côté, tout se passe comme si nous avions un mérite congru à faire valoir, et que le roi se dût, dans le libre don qu'il fait des charges, de favoriser les plus dignes, les plus méritants, les plus capables. Si la monarchie n'est pas une méritocratie, où le mérite puisse avoir un droit objectif et opposable, parce que le roi s'y serait comme contractuellement ou moralement engagé, il convient que le monarque reconnaisse et relève le mérite. L'œuvre de La Bruyère abonde de maximes en ce sens. Il écrit ainsi dans le portrait de Louis XIV, qui clôt le chapitre «Du souverain », qu'il faut, pour régner, quelques dons du ciel comme «une manière de faire des grâces $^{4}$, qui est un second bienfait, le choix des personnes que l'on gratifie $e^{5}$, le discernement des esprits, des talents et des complexions pour la distribution des postes et des emplois; le choix des généraux et des ministres ». Et La Bruyère passe ici insensiblement, comme on le voit, du champ sémantique de la grâce (grâce, gratifié, bienfait) à celui, plus politique, du choix heureux, quoique gratuit. En fait, le monarque doit avoir le mérite supérieur de reconnaitre le mérite d'autrui, et de bien l'employer: "C'est un extrême bonheur pour les peuples quand le prince admet dans sa confiance et choisit pour le ministère ceux même qu'ils auraient voulu lui donner, s'ils en avaient été les maîtres. » Et s'il a lui-même un mérite exceptionnel, il sera la source vivante des mérites qui relèvent les membres de son gouvernement: «Sous un très grand roi, ceux qui tiennent les premières places, n'ont que des devoirs faciles, et que l'on remplit sans nulle peine [...]. Tout coule de source [...]. Ils ont le mérite de subalternes.»

\footnotetext{
4. Nous soulignons.
}

5. Nous soulignons. 
Mais un roi peut quelquefois aussi ne servir de rien qu'à favoriser le moins capable sur le plus capable. Aussi est-ce moins la décision providentielle du roi que le hasard qui signale aux yeux de tous le mérite et le démérite de chacun: "Tout persuadé que je suis que ceux que l'on choisit pour de différents emplois, chacun selon son génie et sa profession, font bien, je me hasarde de dire qu'il se peut faire qu'il y ait au monde plusieurs personnes connues ou inconnues, que l'on n'emploie pas, qui feraient très bien; et je suis induit à ce sentiment par le merveilleux succès de certaines gens que le hasard seul a placés $^{6}$.» Le roi peut même ne pas voir le mérite, et le laisser dans l'ombre sans jamais le relever: «Combien d'hommes admirables et qui avaient de très beaux génies sont morts sans qu'on en ait parlé! Combien vivent encore dont on ne parle point et dont on ne parlera jamais!» Le roi ne manque pas alors à sa justice, mais il manque à sa grandeur. Certes, cela ne saurait décourager ses sujets, car j' ai de toute façon le devoir d'être un bon citoyen et de satisfaire aux obligations de mon emploi, non pas pour obtenir quelque récompense, toujours douteuse, mais pour acquitter la dette que je dois à la république. Je ne suis pas dans la situation d'un coureur qui pourrait refuser de prendre le départ, sous prétexte qu'une fois arrivé premier, il pourrait n'être pas couronné ou récompensé. Ici, je dois de toute façon courir le mieux possible...

On remarquera que le passage précédemment cité se trouve dans le chapitre «Du mérite personnel», où en un sens La Bruyère ne se plaint de rien, puisque, à dire vrai, il n'y a pas matière à se plaindre: "Qui peut, avec les plus rares talents et le plus excellent mérite, n'être pas convaincu de son inutilité, quand il considère qu'il laisse en mourant un monde qui ne se sent pas de sa perte, et où tant de gens se trouvent pour le remplacer»; et pour les premières, dans le chapitre «Du souverain ou De la république», car le roi comme le Grand ne doit avoir qu'une seule consigne: «Sentir le mérite, et quand il est une fois connu, le bien traiter.» Grâce à sa philosophie du mérite, La Bruyère ne critique ainsi fondamentalement rien de la monarchie, lors même qu'il peut dénoncer les manquements du monarque, puisque ces manquements ne sont, pour le souverain, que des manquements à soimême, et moins à sa personne qu'à sa grandeur, et en aucun cas des 
dysfonctionnements du régime ou des injustices envers nous. Il peut flatter la monarchie sans réticence ni visible restriction de pensée, et nous apparaître à proportion comme l'un des plus audacieux critiques de son siècle.

Dans les Caractères, le mérite n'est plus un capital biologique ou historique: «De bien des gens en effet, il n'y a que le nom qui vale quelque chose», nous est-il dit, et ce nom ne suffit plus à faire valoir la personne. Mais le mérite n'est pas encore pour La Bruyère, travail, résultat du travail, force de travailler usée et renouvelée par le travail lui-même, au contraire de Lagneau, pour qui le mérite suit obscurément la logique métaphorique de l'usure (usure du tissu autant que de l'usurier) : il disparait si l'on veut en faire un bien tout fait, mais il nous ouvre continuellement aussi un crédit bien fondé sur des biens à venir. Le mérite, pour La Bruyère, ne serait-ce pas plutôt le principe de ce mouvement silencieux du possible, tout le contraire d'une agitation ou d'une révolte, tout le contraire d'une ambition intéressée, sollicitante, glorieuse? Le mérite, c'est le possible en mouvement, qui ne déplace rien, le seul mouvement que l'on puisse admettre, parce qu'il est moins à la vérité mouvement - contradiction entre ce qui est et ce qui va être, tumulte de ce qui veut être contre ce qui est en place - que la possibilité réelle qu'a et qu'est, aussi bien, toute puissance d'être conduite à l'acte, et d'être ainsi mue; si bien qu'en employant le mérite, on ne substitue pas un autre ordre à l'ordre ancien, mais on rend effectif ce qui existe déjà formellement - et qui ne tend qu'à exister, sans jamais y prétendre.

Il y a plusieurs façons de dépiter un état politique. On peut le critiquer au nom d'une idée rationnelle, ou d'un possible donné par la raison (c'est-à-dire d'un possible nécessaire) comme fondateur ou régulateur: c'est l'idée de pacte social chez Rousseau, de contractus originarius chez Kant, de quasi-contrat chez Léon Bourgeois. Mais on peut aussi bien montrer que si tel pouvoir est légitime, et qu'il est même le meilleur qui soit, il n'assume pas cependant tout le bien qui, pour n'être que possible, ne laisse pas d'être formellement. D'un côté, on critique au nom d'un possible qui n'est que comme une vectorisation ou une restauration idéale de la société; d'un autre côté, on remarque qu'il y a une susceptibilité du possible par le pouvoir qui n'est pas assurée. Le pouvoir n'est pas critiquable, parce que tout pouvoir vient de Dieu (nulla potestas nisi a Deo...); mais la critique est interne au 
pouvoir lui-même, ce qui ne signifie pas autre chose que le pouvoir est en crise, parce que le possible a cette propriété singulière d'être réel, et que si l'homme de mérite est essentiellement silencieux - il n'a rien d'un révolutionnaire réclamant les droits d'une méritocratie contre une aristocratie -, il n'en est pas moins une protestation vivante. L'idée de mérite n'est donc porteuse d'aucune méritocratie - et en ce sens La Bruyère ne prépare pas les philosophes et les mouvements sociaux des siècles qui suivent. Le méritocrate n'est-il pas toujours en effet un peu comme Mopse, qui, lorsqu'on le chasse d'une place destinée à un ministre, s'assied à celle d'un duc et pair, chamboule tout dans la société au nom de la confiance qu'il a en lui-même, et «n'a pas, non plus que le sot, de quoi rougir»? Mais, puisqu'il est de l'essence du mérite de pouvoir n'être pas récompensé, alors même qu'il est moralement nécessaire qu'il le soit, La Bruyère montre que l'idée de mérite, si pertinente dans l'ordre théologique lorsque la providence divine est sans faille, est une idée étrange dès lors qu'elle se trouve exportée dans l'ordre politique, puisque ce n'est plus ici bien souvent que le simple hasard qui place les hommes et distribue les postes. Certes, le roi peut être une nouvelle providence; mais jusqu' où peut s'étendre sa «science des détails » ou sa «diligente attention aux moindres besoins de sa république»? L'idée de mérite critique ainsi moins le pouvoir, qu'elle ne le montre quasi-structurellement en crise, car il y a une exigence, ou bien encore une nécessité morale, disions-nous, d'intussusception du possible qui, pour n'avoir pas d'effet légal, n'est pas un rien, par le droit qui, pour être absolument légitime, n'est pas tout. 